http://jmscr.igmpublication.org/home/ ISSN (e)-2347-176x ISSN (p) 2455-0450

crossref DOI: https://dx.doi.org/10.18535/jmscr/v7i9.10

\title{
A Retrospective Analysis of Toxicity Profile in Pediatric Patients with all Receiving High Dose Methotrexete
}

\author{
Authors \\ Shaik Ruman $^{1 *}$, Umamaheshwar Bairamoni ${ }^{1}$, Himaja Maddela ${ }^{1}$, Mahesh Jakkula ${ }^{1}$, \\ Ganti Manasa', Dr Amatul Ali Sameera ${ }^{2}$ \\ ${ }^{1,2}$ Department of Pharmacy Practice, Sree Dattha Institute of Pharmacy, Hyderabad, India \\ *Corresponding Author \\ Shaik Ruman
}

\begin{abstract}
Aims and Objectives: Our aim was to document the toxicity profile in Acute Lymphoblastic Leukemia in pediatric patients who received only chemotherapeutic agent i.e. High Dose Methotrexate in their consolidation phase of their treatment plan.

Method: A Retrospective study was carried out in the inpatient department of Aware Gleneagles Global Hospital, Hyderabad, India. A total of 100 pediatric in patients with Acute Lymphoblastic Leukemia from the age of 1-18 years in their Consolidation phase consisting of 4 cycles each containing a course of HDMTX from 2017-2018 were included in the study. All information significant to the study was collected from the Patient case sheets and Electronic medical records in the designed patient's proforma or data collection form which includes Patient demographic details, Laboratory values etc. The information was also retrieved by the telephonic conversation with the patient or patient's representative. Results: In our study mean age was found to be $7.07 \pm 4.520$. We found that there was high incidence rate in males (67\%) than females (33). We observed pediatric patients effected with adverse effects such as nausea and Vomiting (25\%), Mucositis (13\%), Neutropenia (5\%), Anemia (55\%), Thrombocytopenia (27\%) and Leucopenia (44\%).
\end{abstract}

Conclusion: Thus from our study we have concluded that toxicity of High Dose Methotrexate can be minimized to great extent by adequate hydration/ Alkalinization, Leucovorin doses, accurate dose of Anti emetics and frequent monitoring of Urine $\mathrm{pH}$, Serum Creatinine, Complete Blood Count as to detect any toxicity and acute management of the toxicity.

Keywords: Acute Lymphoblastic Leukemia, Methotrexate, Toxicity, Febrile Neutropenia, Mucositis.

\section{Introduction}

Acute Lymphoblastic Leukemia (ALL) usually aggressive type of leukemia caused due to malignancy transformation of B-cell or T-cell progenitor cells. ${ }^{[1]}$ It is characterized by the accumulation of lymphoblast's in the marrow, extra medullary sites, peripheral blood etc. frequently accompanied by suppression of normal hematopoiesis. It can also spread to the lymph nodes, spleen, liver, central nervous system (CNS), and other organs. Without treatment, ALL usually progresses quickly. ${ }^{[2]}$

Leukemia's ( $>95 \%$ of which are acute) constitute the most common diagnostic group of childhood cancers worldwide, and in India. Remarkable progress has been made in the treatment of ALL 
(ALL, which constitute $75-80 \%$ of childhood acute leukemia's) with 5-year overall survival rate reaching $90 \%$ in the high-income countries. ${ }^{[3]}$

The treatment involves for children chemotherapy in 4 main phases. ${ }^{[4]}$

- Induction

- Consolidation

- Maintenance

- Re-induction

Whereas, intrathecal chemotherapy is given as to prevent further spread; and also implies with targeted chemotherapy and stem cell transplant depending on the patient condition. ${ }^{[5]}$

\section{Methotrexate}

High-dose methotrexate (HD-MTX), defined as a dose higher than $500 \mathrm{mg} / \mathrm{m}^{2}$, is used to treat a range of adult and childhood cancers. It is a vital component of among many effective chemotherapeutic regimens for childhood ALL.

It is given at doses $12 \mathrm{mg}$ Intrathecal and 20 $\mathrm{mg} / \mathrm{m}^{2}$ orally, intramuscularly, or intravenously as weekly maintenance chemotherapy for ALL to doses as high as $33,000 \mathrm{mg} / \mathrm{m}^{2} \mathrm{IV}$. Doses of 500 $\mathrm{mg} / \mathrm{m}^{2}$ or higher given intravenously are defined as high-dose methotrexate (HD-MTX) and are used to treat a variety of adult and pediatric cancers, including ALL, osteosarcoma, and lymphomas. HD-MTX therapy induces toxicity such as Myelosuppression, Oral mucositis, Acute Renal Failure, Hepatotoxicity, Neurologic toxicity etc. thus may lead to the morbidity, occasional mortality and over all interrupting the cancer therapy. Hence, supportive care is provided such as Continuous Hydration, Alkalinization and Leucovorin rescue etc. Aggressive monitoring of Methotrexate is required regularly as there might be delayed excretion of the drug, increased serum concentration thus causing toxicity. ${ }^{[6]}$

\section{MTX Pharmacology}

Methotrexate, an anti-metabolite agent, inhibits the enzyme Dihydrofolate Reductase mediating the conversion of Dihydro Folic acid to Tetrahydro Folic acid. As being 50,000 times higher affinity for the enzyme than the normal substrate, inhibition is Pseudo irreversible. Tetrahydrofolate is essential for biosynthesis of thymidine and purines, which are needed for synthesis of DNA. Blockade of tetrahydrofolate synthesis by methotrexate leads to inability of cells to divide and to produce proteins. ${ }^{[7]}$

\section{Methodology \\ Plan of Work}

A study was carried out to evaluate toxicity profile in Pediatric patients between 1-18 years with ALL receiving HD-MTX.

\section{Study Site}

The study was conducted in the Oncology department of a tertiary care hospital, Hyderabad in the Indian state of Telangana.

\section{Study Design}

A hospital based retrospective study was carried out on 100 in patients, with ALL patients from the age of 1-18 years who were in their Consolidation phase consisting of 4 cycles each containing a course of HD-MTX from 2018-2019. The data collected included Patient Demographic details (Age, Sex, BMI); Date of admission; Date of discharge; Chief Complaints; Diagnosis; Medical History; Laboratory data related to Hematology, Hepatic, Renal etc; Treatment course involving HD-MTX (Dosage, Route of admission, Frequency, Duration) and Supportive medications such as Leucovorin, Hydration, Alkalization agents etc.

\section{Sample Size}

A total of 100 patients with ALL, who received HD-MTX in their consolidation phase of their treatment protocol of BFM 90; BFM 90 modified/BFM 95.

\section{Sample Period}

The study was carried out for a period of 10 months.

\section{Study Criteria \\ Inclusion Criteria}

- Newly diagnosed patients between 1 to 18 years with ALL receiving HD-MTX in 
their consolidation phase of treatment protocol.

- Patients of either sex.

- Only first 2 cycles, out of 4 cycles of HDMTX consolidation phase was included.

\section{Exclusion Criteria}

- Patients less than 1 year and more than 18 years.

- Patients receiving combinational chemotherapy along with HD-MTX.

- Patients of relapsed ALL.

- Patients who were being treated with a different protocol.

\section{Study Approval}

The study was approved by Ethical Committee of Aware Global hospital, Hyderabad, India.

\section{Statistical Software}

SPSS Software 20.0

\section{Results}

\section{Population Description}

A study was performed with a total of 100 paediatric patients with acute lymphoblastic admitted in the hospital. The Mean Age was 7.07 years and the standard deviation was 4.520 years. Among the study population, 3 (3\%) individuals belonged to infants, age group of 0-2 years, $77(77 \%)$ individuals belonged to children, age group of 3-12 years, 20 (20\%) individuals belonged to adolescents, age group of 13-18 years. Among the study population, 67 (67\%) individuals were found to be males and 33 (33\%) individuals were females.

Among the study population, 49 (49\%) individuals were found to pre B ALL, 33 (33\%) individuals were found to be B ALL and 18 (18\%) individuals were found to be T ALL.

Among the study population of 100 paediatric patients based on column statistical analysis, Pre B ALL were found in $66.7 \%$ of infants, $51.9 \%$ of children and $35 \%$ of adolescents. B ALL in $33.3 \%$ of infants, $32.5 \%$ of children, and $35 \%$ of adolescents. T ALL in $15.6 \%$ of children and $30 \%$ of adolescents.

Among the study population, on the basis of Rome's risk criteria scale there were 62 paediatric patients under Standard risk and 38 patients under High risk.

Figure 01: Bar graph presentation of percentage comparison of vomiting in ALL in each cycle.

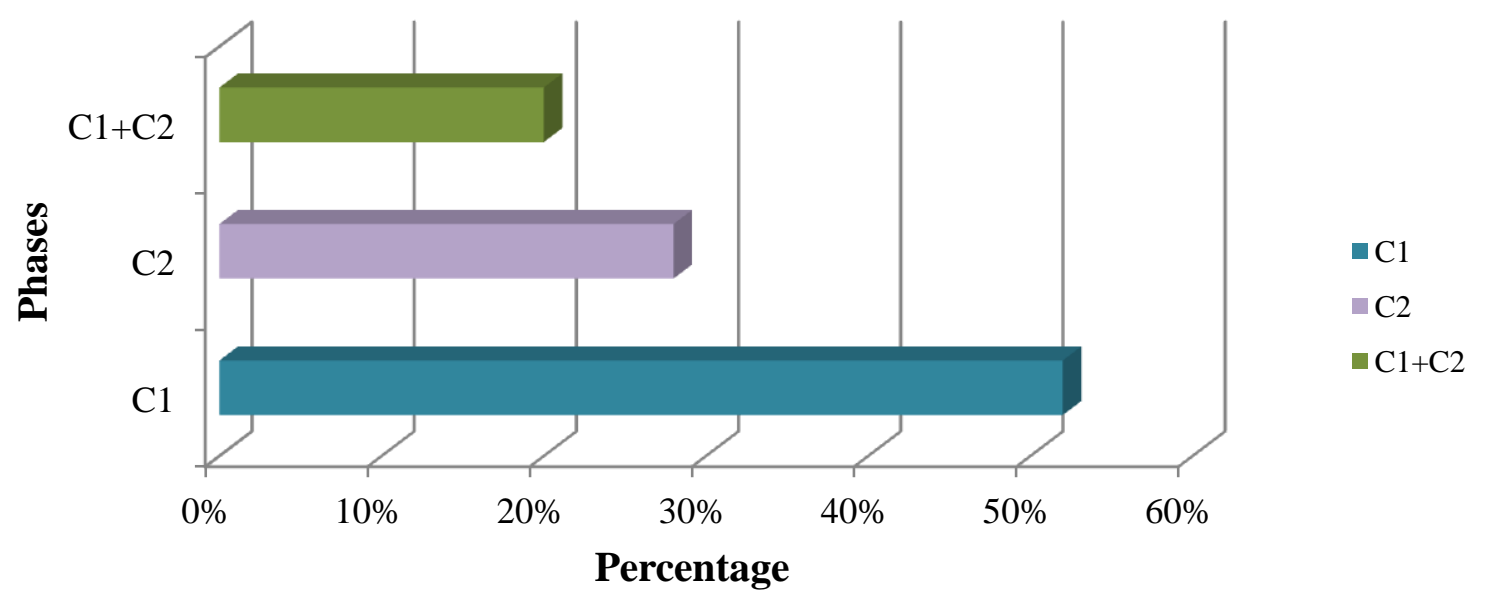

Among the study population, nausea and vomiting was observed in $25 \%$ of patients when considered Cycle (C1) and Cycle (C2) out of which $52 \%$ belong to $\mathrm{C} 1$ and $28 \%$ to $\mathrm{C} 2$ and $20 \%$ both in $(\mathrm{C} 1+\mathrm{C} 2)$ respectively. 
Figure 02: Statistical presentation of age group and percentage of vomiting in ALL by column statistics using chi-square test.

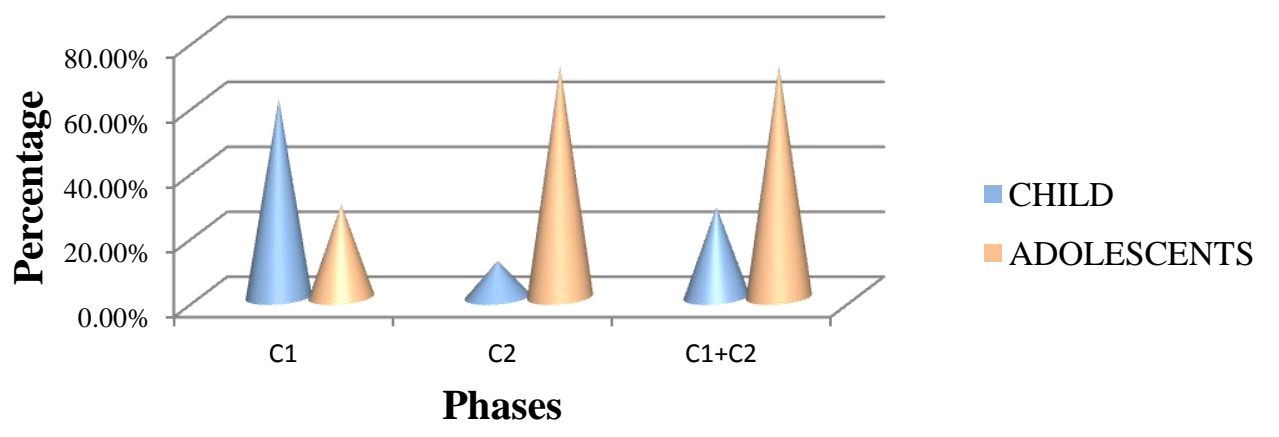

Among the study population based on the statistical presentation of age group and percentage of vomiting in ALL by column statistics, out of 25 patients, there were 18 children and other 5 patients are found to be adolescents.

Figure 03: Column presentation of percentage of drugs used to treat vomiting in ALL.

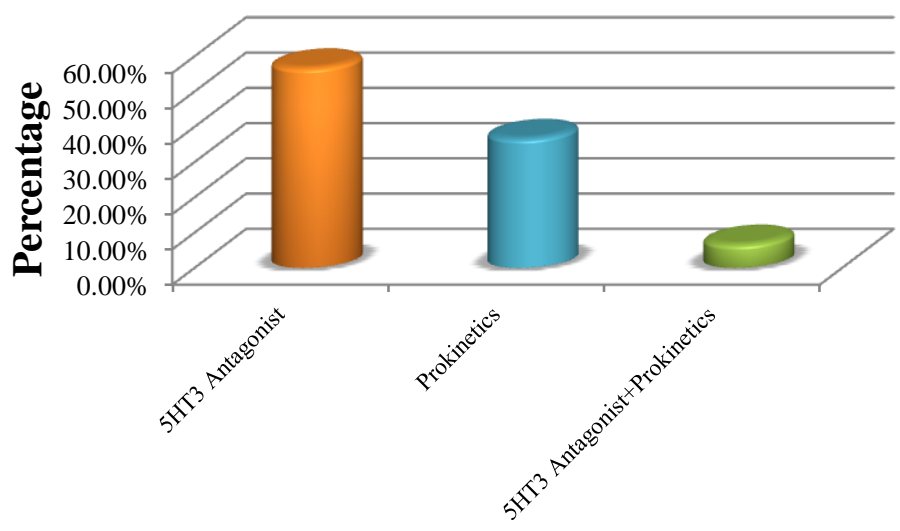

\5HT3 Antagonist

$\square$ Prokinetics

\5HT3 Antagonist+Prokinetics

\section{Class of drug}

Among the study population based on column presentation of percentage of drugs used to treat vomiting in ALL, there was high usage of 5HT3
Antagonist (56.66\%) than Prokinetics (36.67\%); 5HT3 Antagonist + Prokinetics (6.67\%).

Figure 04: Column presentation of grade comparison of mucositis in each cycle.

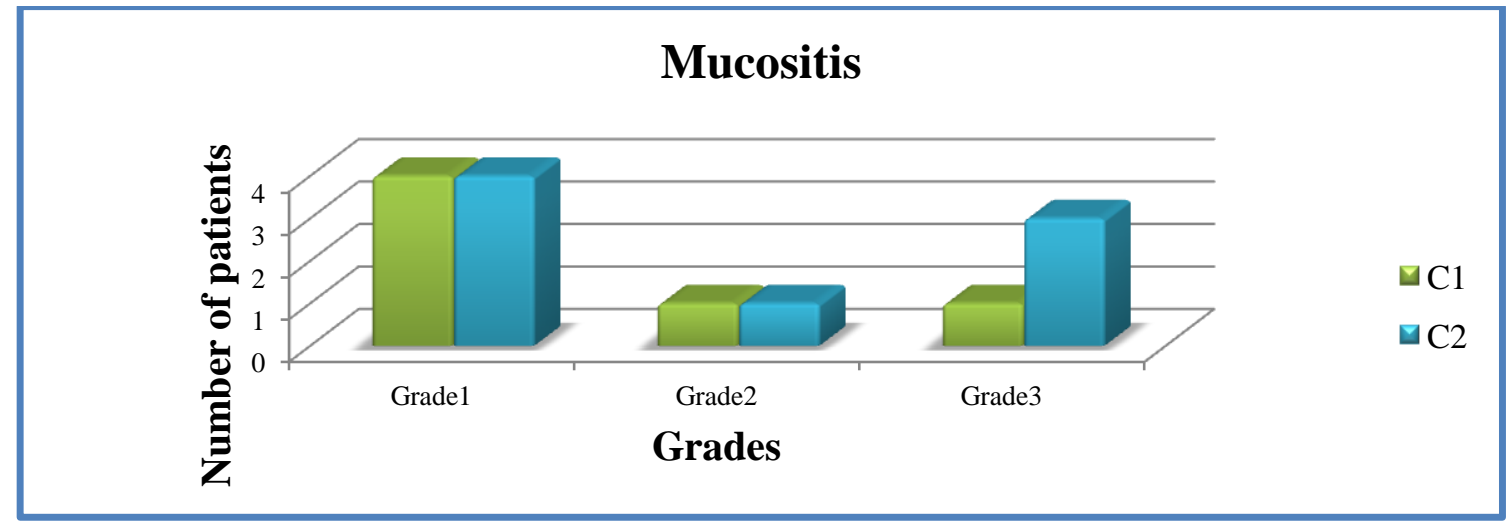

Among the study population mucositis was observed in 14 patients out of which 6 were found in Cycle 1;8 in Cycle 2 respectively. 
Figure 05: Statistical presentation of age group and mucositis grades by column statistics using chi-square test

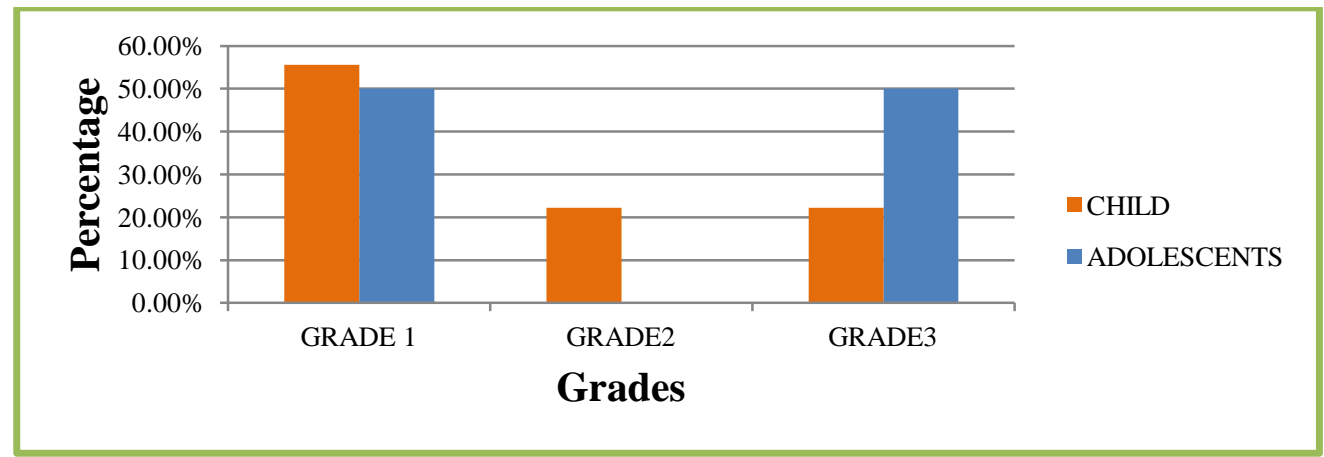

Among the study population, Oral mucositis was found in 13 patients ( 9 were children; 4 were adolescents). In children, 5 patient's fall under Grade 1; 2 under Grade 2; 2 under Grade 3. In adolescents, 2 patient's fall under Grade 1; 2 under Grade 3. Overall there was high incidence of patient's under Grade 1 oral mucositis.

Figure 06: Column presentation of percentage of drugs to treat mucositis in ALL

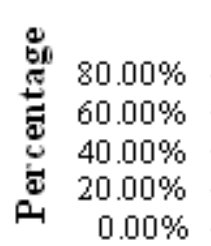

$$
\text { Mo }
$$

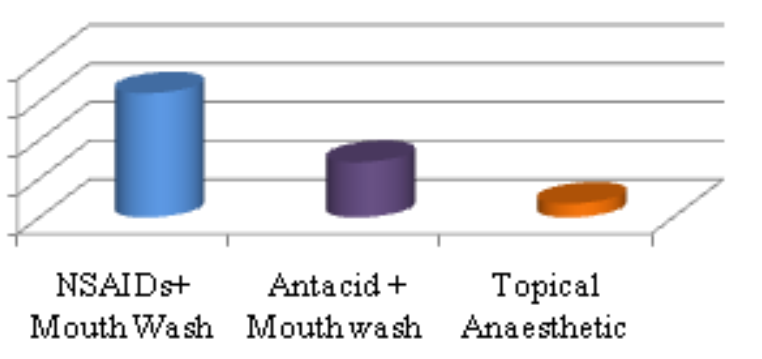

Class of drug
Among the study population based on column presentation of percentage of drugs used to treat mucositis in ALL, there was high usage of
NSAID'S + Mouth wash (64.28\%) than Antacid + Mouth wash $(28.58 \%)$ : Topical anaesthetic $(7.15 \%)$.

Figure 07: Column presentation of percentage of Febrile Neutropenia in ALL.

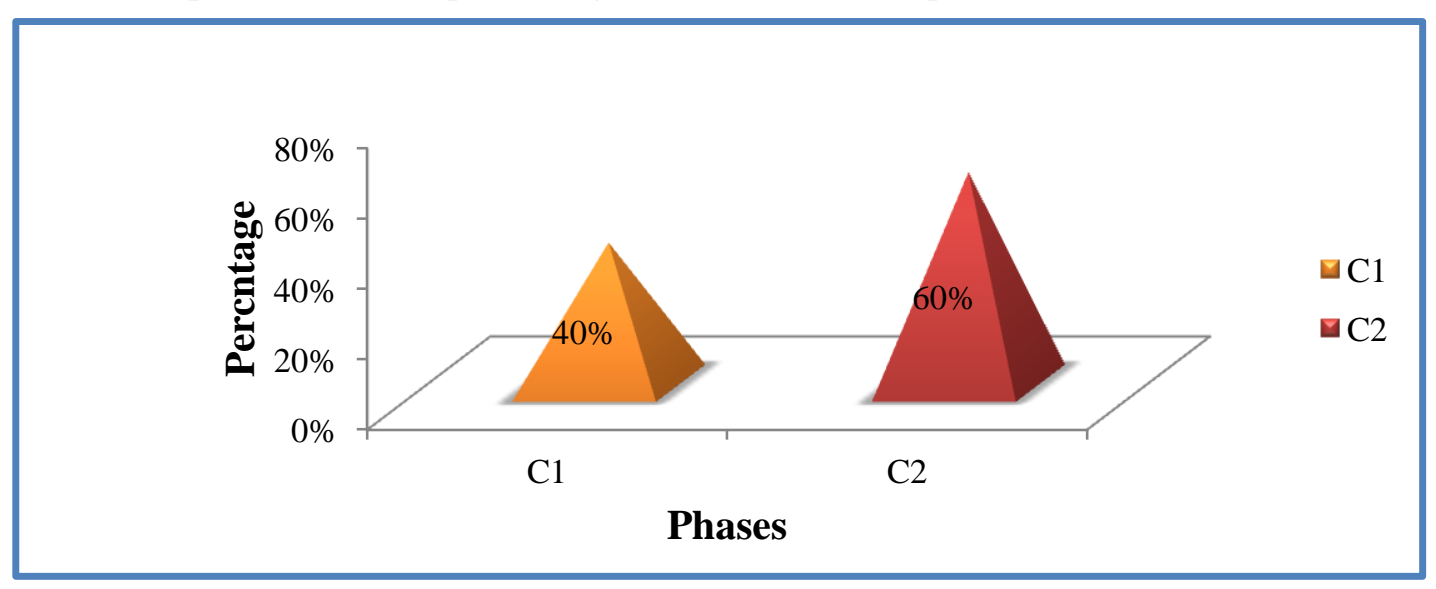

Among the study population, Febrile neutropenia was observed in $5 \%$ of patients when considered
Cycle 1 and Cycle 2 out of which there $2 \%$ patients belong to Cycle 1 and $3 \%$ to Cycle 2 . 
Figure 08: Statistical presentation of age group and febrile neutropenia by column statistics using chi-square test.

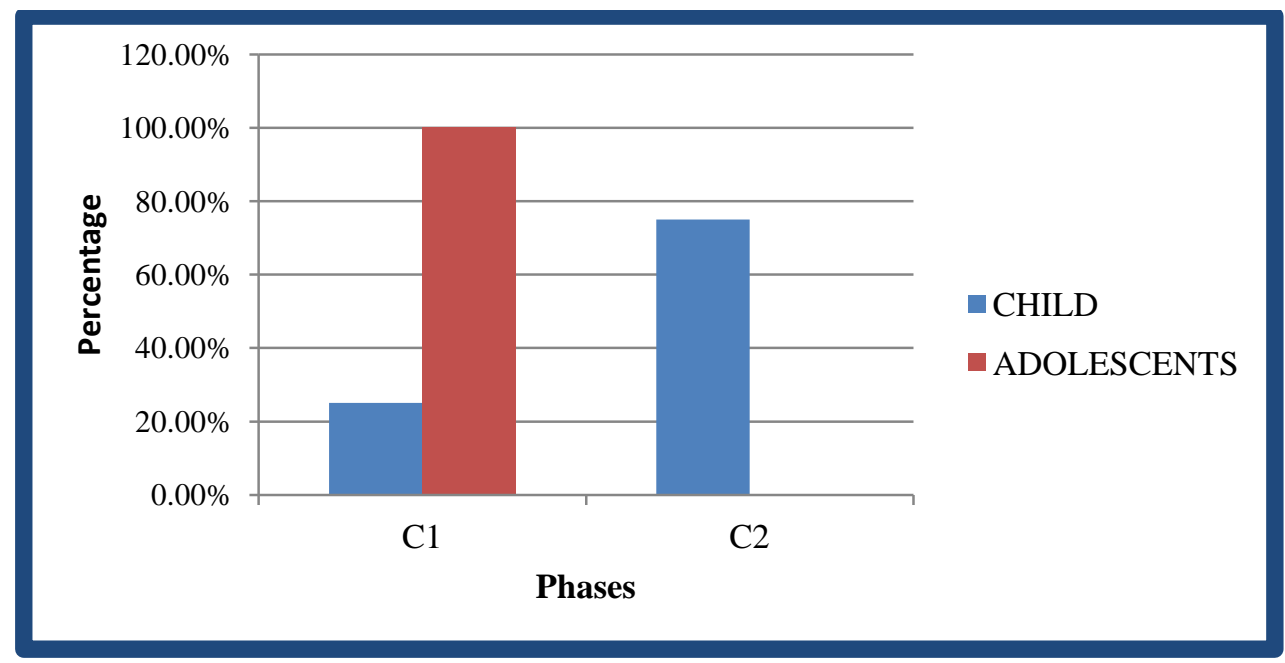

Among the study population, there were 5 cases of (25\%); Grafeel + Tazact (25\%); Magnex forte febrile neutropenia (4 in children, 1 in $(50 \%)$. In adolescents (1 patient) it was treated adolescents). In children (4 patients) febrile with Magnex forte. neutropenia was treated with Grafeel + Cefepime

Figure 09: Column presentation of percentage of drugs used to treat Febrile neutropenia.

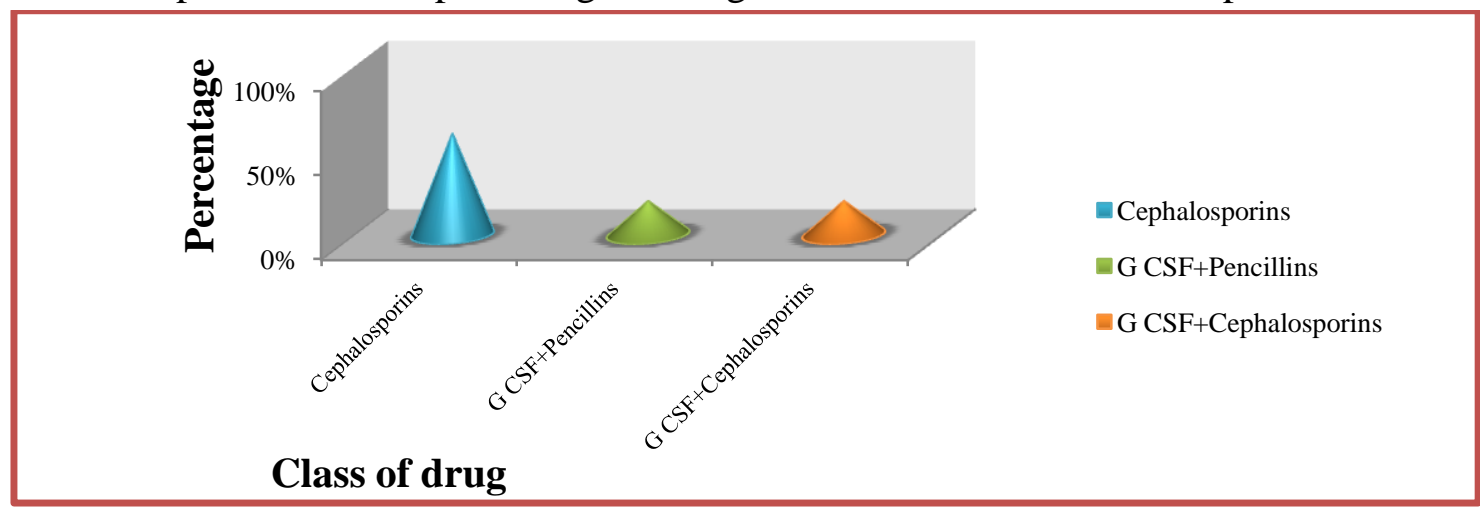

Among the study population based on column Cephalosporin's (60\%) than G CSF + Penicillin's presentation of percentage of drugs used to treat (20\%); G CSF + Cephalosporin's (20\%). Febrile neutropenia there was high usage of

Figure 10: Column graph presentation of percentage of thrombocytopenia in ALL in each cycle

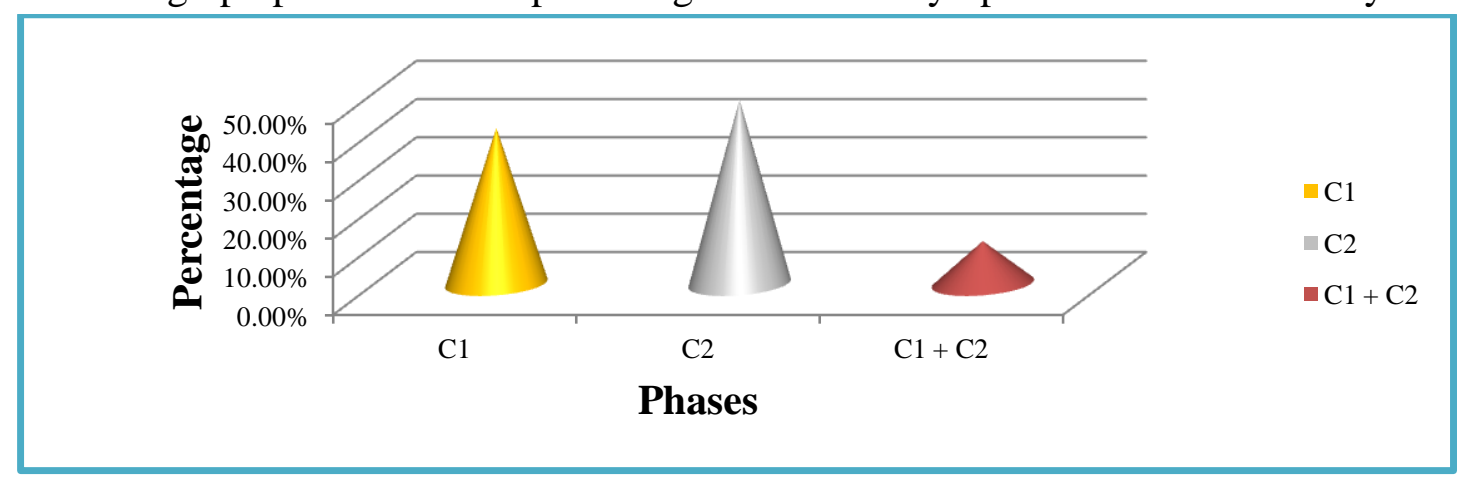

Among the study population, Thrombocytopenia was observed in 27 patients when considered Cycle 1 and Cycle 2 out of which there were 11 patients $(40.74 \%)$ in Cycle 1 and 13 patients $(48.15 \%)$ in Cycle 2; 3 patients $(11.11 \%)$ in $\mathrm{C} 1+\mathrm{C} 2$ respectively. 
Figure 11: Bar graph presentation of percentage of anaemia in ALL in each cycle.

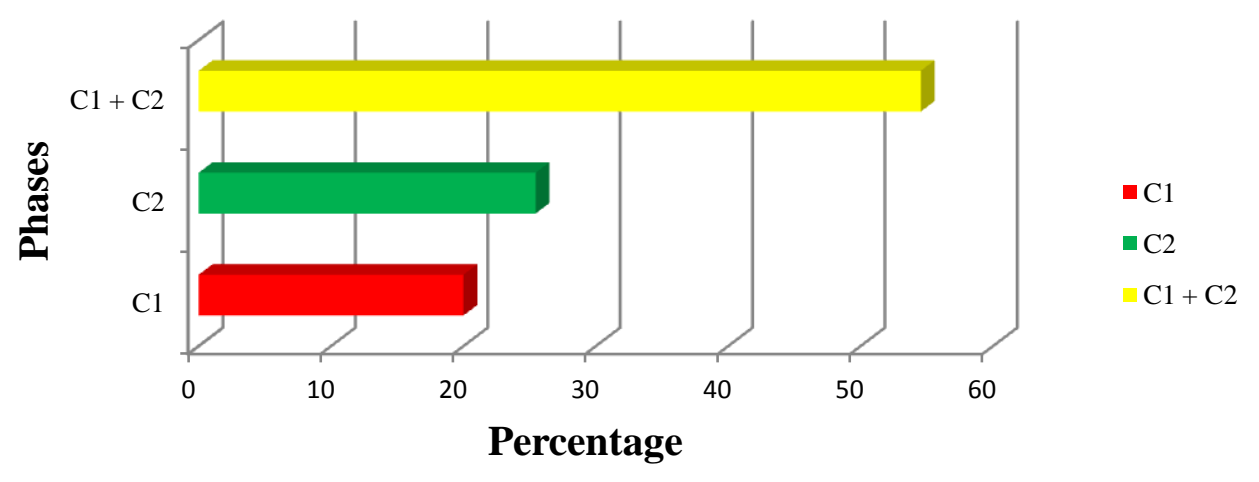

Among the study population, bar graph (20\%) in Cycle 1 and 14 patients $(25.45 \%)$ in presentation of percentage of anaemia in ALL was observed in 55 patients when considered Cycle Cycle 2: 30 patients $(54.55 \%)$ in $\mathrm{C} 1+\mathrm{C} 2$ respectively. 1and Cycle 2 out of which there were 11 patients

Figure 12: Column presentation of percentage of leucopenia in ALL in each cycle

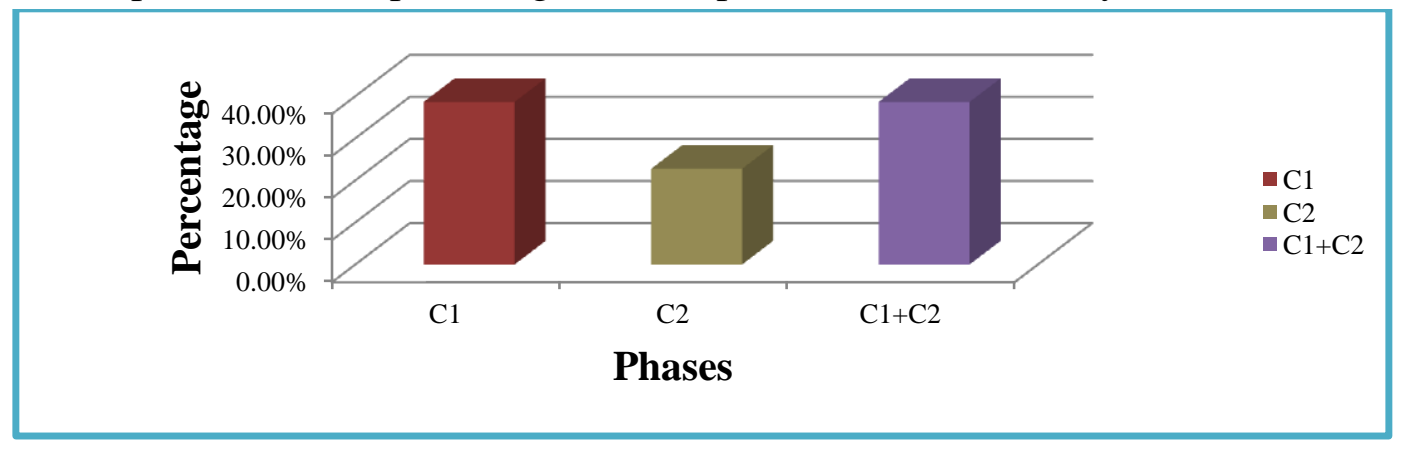

Among the study population according to column presentation of leucopenia in ALL, Leucopenia was observed in 44 patients when considered Cycle 1 and Cycle 2, out of which there were 17 patients $(38.63 \%)$ in Cycle 1 and 10 patients $(22.74 \%)$ in Cycle 2; 17 patients $(38.63 \%)$ were in $\mathrm{C} 1+\mathrm{C} 2$ respectively.

\section{Discussion}

According to the plan of work, pediatric patients were categorized by National Cancer Institute risk criteria scale into the Standard risk and High risk. HD-MTX is the key chemotherapeutic agent in the treatment of ALL which is given by continuous drip of $1 \mathrm{gm}-5.5 \mathrm{gm} / \mathrm{m}^{2}$ in $1000 \mathrm{~mL}$ NS over 24 hours followed by the loading dose of $120 \mathrm{mg}-500 \mathrm{mg} / \mathrm{m}^{2}$ in $100 \mathrm{~mL}$ NS along with Dexamethasone 2-16mg/day, Emeset 2-16mg/day, Hydration $75-200 \mathrm{~mL} / \mathrm{hr}$ by NS depending on individual oncologist's decisions based on patients' clinical status at the time of HD-MTX administration, Alkalinization by 5amp Sodium Bicarbonate and Citralka $2.5-15 \mathrm{~mL} /$ day was adjusted as to keep urine $\mathrm{pH}$ value 7-8 all the time, Leucovorin of 10-20mg of 4-8 doses. Besides, frequent monitoring of Urine $\mathrm{pH}$, Serum creatinine and Complete blood count.

100 pediatric ALL patients in their Consolidation phase (Cycle 1\&2) of BFM treatment protocol were considered containing only HD-MTX chemotherapeutic agent. Based upon WHO classification of ALL, there were Pre B ALL (49), B ALL (33), T ALL (18) patients. The patients were categorized into Infants (1 month-2 years) Children (2-12 years) and Adolescent (12-18 years). Thus Mean age was 7.07 with Standard deviation of 4.520 . In the previous study of Vaishnavi K et al., there were patients of 
ALL/NHL of mean age was $6.8 \pm 3.2$ years out of 53 patients which were B-ALL 25 patients, T- ALL 16 patients, T- NHL 10 patients and relapsed ALL 2 patients. ${ }^{[8]}$ It also corresponds to Traivaree $\boldsymbol{C}$ et al., which included 37 patients of mean age $8.3 \pm 4.2 .{ }^{[9]}$ It also corresponds to the Warrier AR et al., study which included 60 patients of ALL, of mean age 10.57 \pm 7.21 . [10] Another study of Fisgin $\boldsymbol{T}$ et al., recorded 20 patients of ALL of mean age $6.12 \pm 2.91$. $^{\text {[11] }}$ Based upon the National Cancer Institute risk criteria, there were 62 patients of standard risk, and 38 with High risk.

In our study we found higher incidence of males $(67 \%)$ than female $(33 \%)$ patients. This corresponds to Traivaree $\boldsymbol{C}$ et al., where there is $67.6 \%$ females and $32.4 \%$ males. [9] This corresponds with the study of Warrier AR et al., who recorded $58.3 \%$ males and $41.7 \%$ females. ${ }^{[10]}$ It also corresponds to Fisgin $\boldsymbol{T}$ et al., which recorded high incidence of males $(60 \%)$ than females $(40 \%)$. ${ }^{[11]}$

Nausea is one of the most frequent side effects of methotrexate that may adversely impact the adherence and tolerability of this drug. Here, we managed Nausea and vomiting by treating with Dexamethasone, Ondansetron prior 30 minutes of infusion and then post HD-MTX we managed by 5HT3 antagonists (56.6\%), Prokinetics (36.67\%) and combinational of 5HT3 antagonists + Prokinetics $(6.67 \%)$. In our study we have found Nausea in $16 \%$ of children's. It corresponds to Siddique $\boldsymbol{R}$ et al., who observed nausea in $36.6 \%$ of children's (4-10 years) within 48 hours of HDMTX infusion. ${ }^{[12]}$ Another study with reference to Vaishnavi K et al., who observed vomiting in $53 \%$ patients younger than 15 years. ${ }^{[8]}$ It also corresponds to the study of Rask $\boldsymbol{C}$ et al., who observed nausea/vomiting in 28.25 of patients. ${ }^{\text {[13] }}$ Oral mucositis is common major dose limiting toxicity of HD-MTX. It is caused by cellular damage to the rapidly dividing epithelial cells along the GI tract. Here, we managed it by NSAIDs, Mouth wash, Antacids, Topical Anesthetics etc. It was found that there was high use of combination of NSAIDS and Mouth wash in $64.28 \%$ patients and rest were Antacids + Mouth wash (28.58\%), Topical anesthetics $(7.15 \%)$. In our study, HD-MTX was given in the range of $1.5 \mathrm{gm}-6 \mathrm{gm} / \mathrm{m}^{2}$ and Oral mucositis was determined by NCI CTCAE scale and was found in $14 \%$ of patients. It corresponds to Vaishnavi K et al., who observed oral mucositis incidence of $32 \%$ where mean age was $6.8 \pm 3.2$ years. ${ }^{[8]}$ It also corresponds to Fisgin $\boldsymbol{T}$ et al., which recorded $20 \%$ of oral mucositis at a dose of $2 \mathrm{gm} / \mathrm{m}^{2}$ where mean age was $6.12 \pm 2.91$. $^{[11]} \mathrm{It}$ also corresponds with the study of Rask $\boldsymbol{C}$ et al., who observed mucositis in $46.1 \%$ of patients. ${ }^{[13]}$ Febrile neutropenia is life threatening medical emergency condition of infection related fever where neutrophils falls below $<500$ cells $/ \mathrm{mm}^{3}$. Here, we managed it by the use of Cephalosporins, G CSF + Penicillin/ Cephalosporins. It was found that there was high use of Cephalosporins in $60 \%$ and rest was G CSF + Penicillin in $20 \%$ and G CSF + Cephalosporins in $20 \%$ patients. In our study, we have observed five episodes of Febrile neutropenia in cycle 1 and cycle 2. It corresponds to Vaishnavi K et al., who observed febrile neutropenia in $18 \%$ patients younger than 15 years. ${ }^{[8]}$ Another study has been done with reference to Fisgin $\boldsymbol{T}$ et al., where he recorded Febrile neutropenia in 2 patients out of 20 whose age range was $2-13$ years. ${ }^{[11]}$ It also corresponds to Warrier AR et al., who recorded $49.1 \%$ episodes of febrile neutropenia. ${ }^{[10]}$

Anemia, is a condition i.e. lowered ability of blood to carry oxygen as a result of decreased Haemoglobin usually $<12 \mathrm{~g} / \mathrm{dl}$ for women; $<14 \mathrm{~g} / \mathrm{dl}$ for men, which generally follows the diagnosis of the ALL, which gets worsened by HD-MTX due to Myelosuppression. In our study, it was treated by packed cell volume transfusion. In our study, Anemia was observed in $55 \%$ of patients. It corresponds to the study of Vaishnavi $K$ et al., who recorded anemia in $62 \%$ patients. ${ }^{[8]}$ It also corresponds to the study of Fisgin T et al., who recorded anemia in $65 \%$ patients. ${ }^{[11]}$ 
Leucopenia is the lowered number of WBC count caused due to Myelosuppression which is usually below $<4 * 10^{9} / \mathrm{L}$ in blood. Here, we managed it by treatment with Growth colony stimulating factors in the range of $100-300 \mathrm{mcg} /$ day. In our study, leucopenia was found in $44 \%$ patients. It corresponds to Rask C et al., found leucopenia occurring in $61.5 \%$ patients. ${ }^{[13]}$ Another study of Fisgin T et al., who recorded in $65 \%$ patients. ${ }^{[11]}$ Thrombocytopenia, is decrease in platelet count usually below 50,000 cells $/ \mathrm{mm}^{3}$ caused due to Myelosuppression which is managed by Random donor platelet transfusion $(150 \mathrm{~mL})$. In our study it was found to occur in $27 \%$ patients. It corresponds with Rask $\boldsymbol{C}$ et al., who recorded thrombocytopenia in $7.6 \%$ patients. ${ }^{[13]}$ Another study of Vaishnavi K et al., who recorded it in $37 \%$ of patients. ${ }^{[8]}$

\section{Conclusion}

In our present study, we had performed a retrospective analysis of the toxicity profile of HD-MTX drug in pediatric acute lymphoblastic patients.

- In our study we had used National Cancer Institute / Rome Criteria to determine the ALL risk and predict outcome by considering parameters such as Age and WBC. Thereby, it was found that 62 patients fall into Standard risk, and 38 patients into High risk.

- The toxicities evaluated were Oral mucositis, Nausea and Vomiting, Thrombocytopenia, Leucopenia, Febrile Neutropenia, Anemia etc.

- In our study, HD-MTX toxicity were relatively less compared to another study wherein in our study, treatment regimen followed enhanced prophylaxis treatment and monitoring of Methotrexate by Adequate hydration/ Alkalinization, Leucovorin rescue and Antibiotics were given to reduce the toxicity of Methotrexate which was further monitored by Urine $\mathrm{pH}$, Serum Creatinine, CBP etc;
- 5HT3 antagonists such as Ondansetron, and Corticosteroid such as Dexamethasone was given prior infusion of HD-MTX so as to decrease the incidence of acute and delayed nausea and vomiting caused due to HD-MTX.

- In few patients, Oral mucositis was treated either by giving antacid gel or NSAID gel.

- In case of Febrile Neutropenia, it was treated majorly by the use of Antibiotics and then by Colony Growth Stimulating Factor agents etc.

- The anemia, was corrected by Packed cell volume transfusion; Leucopenia by colony growth stimulating factors; Thrombocytopenia by Random donor platelet transfusion.

Thus from our study we have concluded that toxicity of HD-MTX can be minimized to a great extent by adequate hydration/ Alkalinization, Leucovorin doses, accurate dose of Anti emetics and frequent monitoring of Urine $\mathrm{pH}$, Serum Creatinine, Complete Blood Count as to detect any toxicity and acute management of the toxicity.

\section{References}

1. Pui $\mathrm{CH}$, Jeha $\mathrm{S}$ : New therapeutic strategies for the treatment of acute lymphoblastic leukaemia. Nat Rev Drug Discov 6 (2): 149-65, 2007.

2. Peterson LC, Bloomfield CD, Brunning $\mathrm{RD}$ : Blast crisis as an initial or terminal manifestation of chronic myeloid leukemia: a study of 28 patients. Am J Med 60(2): 209-220, 1976.

3. Pui CH, Yang JJ, Hunger SP, Pieters R, Schrappe M, Biondi A, et al. Childhood ALL: Progress through collaboration. J ClinOncol. 2015;33:2938-48.

4. Dipiro. Chapter 142: Acute leukemia. Gary C. Yee Editor. In: pharmacotherapy A pathophysiologic approach; $8^{\text {th }}$ Edition .P.2384.

5. Perez-Andreu V, Roberts KG, Harvey RC, et al. Inherited GATA3 variants are 
associated with Ph-like childhood ALL and risk of relapse. Nat Genet 2013; 45:1494-1498

6. Mantadakis E, Cole PD, Kamen BA. High- dose methotrexate in ALL: where is the evidence for its continued use? Pharmacotherapy: The Journal of Human Pharmacology and Drug Therapy. 2005 May;25(5):748-55.

7. Tripathi KD. Essentials of MEDICAL PHARMACOLOGY. 2013;7:862

8. Vaishnavi K, Bansal D, Trehan A, Jain R, Attri SV. Improving the safety of high- dose methotrexate for children with hematologic cancers in settings without access to MTX levels using extended hydration and additional leucovorin. Pediatric blood \& cancer. 2018 Dec;65(12):e27241.

9. Traivaree C, Likasitthananon N, Monsereenusorn C, Rujkijyanont P. The effect of intravenous hydration strategy on plasma methotrexate clearance during intravenous high-dose methotrexate administration in pediatric oncology patients. Cancer Management and Research. 2018;10:4471.

10. Warrier AR, Rejiv R, Biswajit D, Ramanan SG, Sagar TG. High-dose methotrexate in the treatment of ALL: Toxicity profile and comparison of tolerability betwen two dosage schedules. Journal of Clinical Oncology. 2010 May 20;28(15_suppl):9573-.

11. Fisgin T, Yarali N, Kara A, Bozkurt C, Birgen D, Erten U, Duru F. Hemostatic side effects of high-dose methotrexate in childhood ALL. 2004;21(1):77-83.

12. Siddique R, Hafiz MG, Jamal CY, Karim MA, Islam A, Alia RA. Randomized Double Blind Trial to Compare the Efficacy of Granisetron And Ondansetron in Controlling Emesis in Children with ALL. Bangladesh Journal of Child Health. 2012;36(3):115-21.
13. Rask C, Albertioni F, Bentzen SM, Schroeder H, Peterson C. Clinical and pharmacokinetic risk factors for high-dose methotrexate-induced toxicity in children with ALL: a logistic regression analysis. Acta Oncologica. 1998 Jan 1;37(3):27784. 